\title{
Intervenções educativas de acolhimento à novos colaboradores: 0 papel do enfermeiro e instrutores internos
}

\section{Educational interventions to welcome new employees: the role of nurses and internal instructors}

${ }^{1}$ Sebastião Ezequiel Vieira ezequielvvieira@hotmail.com

${ }^{1}$ Marcelo Paraíso Alves

${ }^{2}$ Gisele de Souza Silva

${ }^{2}$ Luciana de Freitas Souza

${ }^{2}$ Tatiane de Assis Bernardo Vieira

\section{RESUMO}

Nas últimas décadas, grandes mudanças têm ocorrido no cenário da educação hospitalar. Este fato pode ser observado pelo uso de métodos inovadores e cada vez mais presentes no treinamento e desenvolvimento de profissionais desta área. A preocupação principal das instituições tem se voltado para o aprimoramento profissional, tendo em vista a necessidade crescente de melhorias no atendimento aos pacientes, sendo necessário o desenvolvimento de ações que apresentam como objetivo principal capacitar e desenvolver os profissionais da saúde, para realizarem a assistência de Enfermagem com qualidade e segurança. Neste contexto, o processo de qualificação e aprimoramento profissional é ressaltado, neste trabalho, como um dos pontos altos para o bom desempenho dos profissionais na área de saúde. Como objetivo principal a presente pesquisa procura descrever a atuação dos enfermeiros do setor de Treinamento e Desenvolvimento na capacitação dos instrutores internos na estratégia de treinamento e acolhimento dos novos profissionais de Enfermagem admitidos na Fundação Cristiano Varella. O estudo tem caráter quantitativo e utilizou-se de um questionário semiestruturado que foi aplicado a 48 profissionais técnicos de Enfermagem que atuam na referida instituição. Os resultados demonstraram que a estratégia de capacitação e acolhimento dos profissionais admitidos por meio dos instrutores internos foi uma ação bem elaborada e que apresentou excelentes resultados para equipe de Enfermagem.

Palavras-chave: Educação permanente. Acolhimento. Enfermeiro e instrutores internos.

\section{ABSTRACT}

In recent decades, great changes have taken place in the hospital education scenario. This can be observed through the use of innovative methods and increasingly present in the training and development of professionals in this area. The main concerns of the institutions have turned to professional improvement, in view of the growing need for improvements in patient care. Requiring the development of actions that have as main objective to train and develop health care professionals, to be performing nursing care with quality and safety. Within this context, the qualification and professional development process is highlighted in this work as one of the highlights for the good performance of professionals in the health area. The main objective of the present study seeks to describe the activities of nurses Training and Development sector in the training of internal instructors in the training strategy and welcoming new nurses admitted to the Cristiano Varella Foundation. This study is quantitative approach, where we used a semi-structured questionnaire in field research, which is applied to 48 technical nursing professionals who work in this institution. The results showed that the training strategy and reception of employees hired through internal instructors was an elaborate action and presented excellent results for the nursing staff.

Keywords: Continuing Education. Welcome. Nurse e internal instructors.

1 Centro Universitário de Volta Redonda - UniFOA

2 Fundação Cristiano Varella, Muriaé, MG 


\section{INTRODUÇÃO}

A presente pesquisa é parte do trabalho de conclusão de curso desenvolvido no ano de 2015 como integrante para conclusão do curso de Bacharel em Enfermagem. A pesquisa foi desenvolvida em um Hospital do município de Muriaé, Minas Gerais, Fundação Cristiano Varella com intenção de descrever a atuação dos enfermeiros do setor de Treinamento e Desenvolvimento na capacitação dos instrutores internos na estratégia de treinamento e acolhimento dos novos profissionais de enfermagem.

Segundo Tachizawa e colaboradores (2006), o treinamento dos profissionais dentro das instituições tem como objetivo proporcionar um adequado preparo técnico-científico, o que, por sua vez, lhes fornece condições de se manterem atualizados e aptos para executar suas atividades. Outro benefício proporcionado pelo treinamento é a capacidade de os profissionais aperfeiçoarem os resultados de forma contínua levando-os ao processo de desenvolvimento profissional.

No que se refere ao desenvolvimento profissional, este se caracteriza por ser um método de ensino aplicado em um período mais prolongado, atuando de forma positiva nas habilidades dos profissionais, despertando as motivações necessárias que os levem a um processo de renovação e melhoria de suas atividades. Desta forma, tornam-se profissionais indispensáveis para instituição (MILKOVICH; BOURDREAU, 2010).

Neste contexto, a presente pesquisa objetiva descrever a atuação dos enfermeiros do setor de Treinamento e Desenvolvimento na capacitação dos instrutores internos na estratégia de treinamento e acolhimento dos novos profissionais de Enfermagem admitidos na Fundação Cristiano Varella.

\section{REVISÃO DA LITERATURA}

Segundo Schonberger (1996), o responsável por iniciar as ideias correspondentes às necessidades de treinamento foi Frederick W. Taylor. Este observou que os profissionais necessitavam ser capacitados para realizar suas funções com efetividade, desta forma pôde ser considerado o pai do treinamento.

No começo do século XX, os programas de treinamento foram influenciados pela Escola Clássica de Administração, com objetivos direcionados para preparar os profissionais a alcançar os níveis máximos de produção. A Escola de Relações Humanas apresentou inovações e o treinamento deixou de ter o foco somente na produção do indivíduo e passou a considerar os aspectos psicossociais dos profissionais. Sendo assim, os programas de treinamento nas organizações iniciaram um novo método de capacitação dos profissionais para exercerem suas atividades, considerando ações direcionadas ao relacionamento interpessoal e sua integração à organização (GIL, 2006).

Para Chiavenato (2010), o treinamento pode ser considerado como uma ação didática, realizada de forma criteriosa e coordenada, pela qual os indivíduos adquirem domínio, competência em busca de propósitos estabelecidos.

Com a utilização do treinamento como ferramenta de qualificação profissional, as instituições apresentam melhores resultados, uma vez que por consequência do treinamento haverá maior produtividade e qualidade da assistência prestada, trazendo benefícios para a empresa e para o indivíduo que adquiri novos conceitos, novas habilidades, que será primordial para sua formação profissional.

Em relação ao desenvolvimento, Pio (2006) o define como uma soma de ações com a finalidade de conhecer a capacidade das pessoas em relação ao aprendizado e a produtividade, objetivando a transformação de condutas e iniciativas dos profissionais. Tem o propósito de ampliar as práticas e a motivação dos profissionais ocasionando um bom clima organizacional. 
Percebe-se que o desenvolvimento não engloba somente ações para capacitar os profissionais para desenvolver suas funções através de novos conhecimentos e habilidades, mas também possibilita o aprendizado para modificar antigos hábitos ou comportamentos, objetivando não somente o processo de capacitação, mas também o processo educativo.

A educação é considerada uma atribuição humana primordial para a manutenção e adequado funcionamento de uma sociedade bem-sucedida. A sociedade como um todo deve garantir a formação de seus integrantes, desenvolver suas habilidades para que eles possam contribuir com ações inovadoras no meio em que estão inseridos (MEIRER et al., 2006). No âmbito da sociedade, destaca-se relevante importância ao processo educativo na saúde.

Tendo em vista essa real necessidade de formação e desenvolvimento dos profissionais de saúde, a gestão de recursos humanos apresenta componentes essenciais para assegurar a eficiência, eficácia e efetividade das ações dos serviços de saúde através dos programas de educação continuada e educação permanente.

Conforme Paschoal (2004), a educação continuada ocorre após a profissionalização, são atividades desenvolvidas com objetivo de proporcionar renovação dos conhecimentos e aquisição de novas informações e atividades, são embasadas em metodologias formais com durabilidade definida.

Já a educação permanente é a associação entre a formação e realização do trabalho, está alicerçada na aprendizagem e acontece a partir da problematização diária que se apresenta na atuação profissional, considerando os saberes e vivências já existentes da equipe (BRASIL, 2004).

Nesta pesquisa, optou-se por trabalhar com a terminologia de educação permanente tendo em vista as características apresentadas pelo objeto da pesquisa, sendo eles os instrutores internos e o profissional enfermeiro. Desta forma, tem-se um processo de educação menos formal e multiprofissional inserido na rotina de serviço dos profissionais citados.

Em relação aos profissionais alvos da pesquisa, o profissional enfermeiro possui graduação de nível superior intitulando-se bacharel em Enfermagem, o que permite a esse profissional atuar nas mais diversas áreas com destaque a atenção primária nas unidades básicas de saúde e na atenção secundária e terciária nos hospitais de médio e grande porte. Este profissional pode atuar diretamente na assistência ao paciente e em setores administrativos. Por outro lado, é crescente a atuação do enfermeiro na capacitação e propagação de conhecimento frente à equipe de saúde.

O enfermeiro que atua no processo de educação permanente é considerado um líder que desempenha o papel de educador. Tendo este á responsabilidade de capacitar a equipe de Enfermagem, tornando os profissionais habilitados a prestar uma assistência de qualidade sempre com base nos conhecimentos técnicos e científicos, o que impacta diretamente na qualidade da assistência prestada (BARBOSA; SAMPAIO, 2003).

Nesta pesquisa foi possível observar a função do enfermeiro no processo de educação não só nos treinamentos, bem como no desenvolvimento de outros profissionais para replicação do conhecimento.

Dentro da equipe de Enfermagem nos âmbitos da Fundação Cristiano Varella, os instrutores internos são profissionais técnicos de Enfermagem que passam por uma capacitação junto ao setor de treinamento e desenvolvimento da instituição.

O processo de desenvolvimento dos instrutores ocorre por meio de ações específicas voltadas para aprimoramento de técnicas de ensino e acolhimento dos profissionais admitidos na instituição, através de reuniões em que se pontuam características e especificidades a serem trabalhadas nos treinamentos dos colaboradores; 
posteriormente é iniciado o processo de acompanhamento dos instrutores em sua prática ensino. Nestes momentos, o enfermeiro do setor de treinamento passa a realizar visitas semanais nas unidades onde ocorre o processo.

O processo de acolhimento ocorre no próprio setor onde os profissionais instrutores atuam. Os treinamentos realizados pelos instrutores acontecem da seguinte forma: inicialmente acolhem os novos no setor, ficando sob sua responsabilidade direcionar este colaborador dentro dos âmbitos da unidade. Na sequência, o instrutor é incumbido de repassar alguns treinamentos específicos para o novo colaborador. Neste contexto, outro ponto importante desenvolvido pelo instrutor interno dentro do setor é o processo de acolhimento dos novos colaboradores. Desta forma, o instrutor passa a ter responsabilidade de acompanhá-lo durante toda a fase de adaptação dentro do setor. Esta ação tinha como objetivo minimizar os receios, incertezas e as dificuldades encontradas pelos novos colaboradores no processo de adaptação.

Mediante o cenário que se apresenta, observa-se, na atuação do instrutor interno, a aplicação do processo de educação permanente que conforme descrito na pesquisa é constituído por ações que capacitam os profissionais no próprio ambiente de trabalho. O que em geral é o local onde ocorre a problematização decorrente da atuação do profissional, assim é conferido ao colaborador a possibilidade de adquirir características que vão além do conhecimento técnico, possibilitando a interação com a equipe, autonomia e a relação da teoria com a prática.

\section{MATERIAL E MÉTODO}

A pesquisa foi desenvolvida no Hospital do Câncer de Muriaé - MG Fundação Cristiano Varella.

O estudo foi realizado no período de maio a setembro de 2015, com uma amostra composta por 50 profissionais técnicos de Enfermagem entre eles 20 instrutores internos e 30 profissionais auxiliares e técnicos de Enfermagem que foram capacitados por eles. Houve a exclusão de 2 profissionais decorrente de licença médica sendo assim a amostra final contou com 48 profissionais.

Trata-se de uma pesquisa de cunho quantitativo com questões específicas relacionadas ao tema da pesquisa. Segundo o Richardson (1999), a pesquisa quantitativa apresenta características como a utilização da quantificação da coleta de dados e do tratamento dos mesmos através de métodos estatísticos.

De acordo com Minayo (2007), o método quantitativo tem como objetivo fornecer os dados, indicadores e tendências observáveis, produzindo modelos teóricos de alta abstração com aplicabilidade prática.

Este trabalho teve como base a análise dos dados coletados por meio de questionário aplicado aos profissionais envolvidos com questões específicas relacionadas ao tema da pesquisa. Foram utilizados dois questionários auto elaborados, semiestruturados (ANEXO A e B), cada questionário contendo 04 questões fechadas. Os participantes assinaram um Termo de Consentimento Livre e Esclarecido, Resolução 196/96 (ANEXO C), indicando sua participação na pesquisa por livre e espontânea vontade, permitindo assim a divulgação dos dados coletados.

\section{RESULTADOS E DISCUSSÃO}

Inicialmente os dados analisados foram as variáveis de caracterização dos colaboradores. A amostra foi composta por 48 profissionais técnicos de Enfermagem dentre eles, 37,5\% instrutores internos que estão inseridos no Projeto de Instrutores Internos e 62,5\% profissionais capacitados pelos instrutores que responderam ao questionário semiestruturado. 
A amostra foi composta principalmente por profissionais do sexo feminino $83 \%$ contra $17 \%$ de profissionais do sexo masculino, a faixa etária predominante foi de 30 anos (67\%), e o de tempo de trabalho na empresa predominou de 3 a 6 anos (36\%).

A seguir é apresentada a análise dos dados relacionados aos questionários aplicados aos instrutores internos e profissionais admitidos. Foi se perguntado como avaliavam o trabalho desenvolvido pelo enfermeiro do setor de treinamento e desenvolvimento e ao analisar a resposta dos entrevistados, observou-se que, na opinião dos instrutores internos, $78 \%$ analisaram como ótimo e $22 \%$ como bom, para os profissionais admitidos $70 \%$ analisaram como ótimo, 27\% como bom e 3\% como regular. Pode-se observar que, em sua maioria, os profissionais avaliaram como ótimo a atuação do profissional enfermeiro junto à equipe de Enfermagem através do setor de treinamento e desenvolvimento, o que demonstra a relevância de sua atuação educativa e reforça a opinião do autor Silva (2009) que relata que o enfermeiro é essencial na integração e na efetividade dos setores de educação no ambiente hospitalar, pois é um profissional que tem conhecimento da realidade do processo de trabalho, apresenta habilidades técnicas, científicas, éticas e é capaz de incentivar a equipe de enfermagem na participação das atividades propostas.

Quando perguntado aos participantes da pesquisa sobre avaliação que faziam quanto à estratégia de acolhimento e capacitação dos profissionais admitidos, percebe-se que conforme avaliação dos instrutores internos, $73 \%$ avaliaram como ótimo e 27\% como bom. Já para os profissionais admitidos, 70\% avaliaram como ótimo, $27 \%$ bom e $3 \%$ regular. Através da avaliação dos profissionais, que em sua maioria avaliaram a estratégia como ótimo, é possível afirmar que foi uma iniciativa que apresentou impacto positivo na equipe, possibilitando aos novos profissionais e aos próprios instrutores, conhecimento e habilidades importantes para estarem atuando juntos na assistência dos pacientes.

Desta forma, a educação permanente pode ser aplicada como uma estratégia de preparação para os profissionais, o que significa fortalecimento de diferentes concepções e práticas de capacitação, envolvendo o conhecimento as atividades diárias das instituições e incentivando os trabalhadores a atuarem no processo educativo (BRASIL, 2009).

Perguntou-se aos instrutores internos e aos profissionais admitidos sobre o desempenho que os novos profissionais apresentaram após passarem pelo processo de acolhimento e capacitação realizado pelos instrutores e pelo enfermeiro do setor de treinamento e 56\% dos instrutores internos avaliaram o desempenho dos profissionais como ótimo e 44\% como bom. Para os profissionais admitidos, 54\% avaliaram como ótimo, 43\% bom e 3\% regular. Os dados mostram que após a implementação do projeto, instrutores internos e profissionais admitidos, em sua maioria, julgaram que o desenvolvimento dos novos profissionais apresenta melhor desempenho após passarem pela experiência de serem treinados e acolhidos pelos instrutores internos. Quando se relaciona a opinião dos entrevistados com a literatura, Peduzzi (2009) afirma que a educação permanente confere aos profissionais habilidades como comunicação e interação com a equipe em que está inserido. Isso gera vínculo entre os profissionais experientes e novos, proporcionando desenvolvimento de ações a serem realizadas no local de trabalho e equipe.

No que ser refere à atividade de instrutor interno, foi questionado aos colaboradores se esta nova atribuição contribuiu para o crescimento profissional dos mesmos. Pode-se observar excelente adesão por parte dos instrutores internos em relação à estratégia proposta, pois 100\% dos colaboradores concordaram que sua participação do processo de acolhimento e capacitação aos novos colaboradores como instrutores internos lhes proporciona desenvolvimento profissional. Segundo Oliveira e colaboradores (2011), a inserção da educação permanente nas instituições hospitalares é considerada um método para alcançar o desenvolvimento profissional, pois conduz o profissional a adquirir princípios como: competência profissional, motivação e contínua avaliação, características que objetivam uma atuação de forma crítica e reflexiva.

Outro ponto de grande relevância para pesquisa foi a avaliação dos novos colaboradores quanto à atividade desenvolvida pelos instrutores internos. Perguntou-se: como avaliam as atividades desempenhadas pelos ins- 
trutores internos. os profissionais admitidos avaliaram a atribuição do instrutor interno da seguinte forma: 77\% como ótimo e $23 \%$ como bom. Observa-se um resultado muito positivo no desenvolvimento de suas funções. Assim como descrito na pesquisa, os instrutores internos recebem treinamentos e capacitações por intermédio do enfermeiro do setor de treinamento e desenvolvimento. A partir daí são responsáveis por multiplicar esse conhecimento e acolher os novos profissionais do setor. O objetivo principal é que os profissionais que adentram na instituição atuem com qualidade e segurança. Mediante os dados apresentados, é razoável concluir que os instrutores internos estão sendo eficazes em sua atuação, pois foram bem avaliados pelos novos profissionais. Assim, é possível ressaltar Silva (2006) ao descrever que desenvolver pessoas não é somente conceder informações para adquirir novas habilidades e atitudes para ser eficiente nas ações realizadas, mas também ensinar informações básicas para que possam obter novas ideias, concepções, mudanças de hábitos e condutas para se tornarem mais eficientes nas suas atividades.

\section{CONCLUSÃO}

A presente pesquisa buscou avaliar a importância de ações educativas nos âmbitos dos serviços de Enfermagem, mais especificamente a estratégia de acolhimento e capacitação aplicada aos profissionais admitidos. Abordaram-se assuntos pertinentes ao processo de educação, dando ênfase a educação permanente que é um dos métodos de educação mais adequados para o ambiente de trabalho onde as dificuldades são apresentadas e surgem as dúvidas relacionadas ao desempenho das funções a serem executadas.

Outro aspecto analisado foi a importância da implementação de estratégias de capacitação para equipe de saúde, a atuação do enfermeiro do setor de treinamento e desenvolvimento e dos instrutores internos como educadores e multiplicadores de conhecimento. Mediante a aplicação da estratégia de acolhimento e capacitação dos profissionais admitidos, foi possível visualizar resultados satisfatórios como já demonstrado na pesquisa, sendo ressaltada a relevância do profissional enfermeiro no desenvolvimento da estratégia, preparando os instrutores internos para aturarem junto aos profissionais admitidos.

Vale destacar a avaliação positiva de desempenho dos colaboradores que passaram pelo processo de acolhimento dos instrutores internos, estes apresentaram melhoria no desempenho profissional.

Desta forma, torna-se pertinente concluir que as intervenções de acolhimento e capacitação dos novos colaboradores implantado na instituição FCV apresentou excelentes resultados, melhorando o desempenho dos profissionais admitidos e proporcionando um ambiente mais favorável ao aprendizado e capacitação profissional. O presente trabalho reforça a importância do desenvolvimento ações educativas que busquem o aperfeiçoamento da equipe de saúde no ambiente hospitalar, espera-se que este trabalho traga reflexões e estimulo a pesquisas na área de educação hospitalar. 


\section{REFERÊNCIAS}

BARBOSA, R.C.; SAMPAIO, C.E.P. Educação permanente em saúde no controle de infecção hospitalar em terapia intensiva. Enfermagem Atual, Rio de Janeiro, v. 3, n.16, p. 14-18, jul./ago. 2003.

BRASIL. Ministério da Saúde. Secretaria de Gestão do Trabalho e da Educação na Saúde. Departamento de Gestão da Educação em Saúde. Política Nacional de Educação Permanente em Saúde. Brasília, 2009. Disponível em: <http://bvsms.saude.gov.br/bvs/publicacoes/politica_nacional_educacao_permanente_saude.pdf >. Acesso em: 25 ago. 2015.

CHIAVENATO. I. Gestão de pessoas. 3 ed. Rio de Janeiro: Elsevier, 2010.

GIL, A. C. Gestão de pessoas: Enfoque nos papéis profissionais. São Paulo: Atlas, 2006.

MEIRER, Marineli Joaquim; MANTOVANI, Maria de Fátima; PASCHOAL, Amarílis Schiavon. Percepção da educação permanente, continuada e em serviço para enfermeiros de um hospital ensino. Revista da Escola de Enfermagem USP, São Paulo, v. 41. n.3. p. 479, 2006. Disponível em: $\leq$ http://www.scielo.br/pdf/reeusp/ v41n3/19.pdf>. Acesso em: 14 jul. 2015.

MILKOVICH, George T.; BOUDREAU, John W. Administração de Recursos Humanos. São Paulo: Atlas, 2010.

MINAYO, M. C. S. O desafio do conhecimento: pesquisa qualitativa em saúde. 2. ed. São Paulo: HucitecAbrasco, 2007.

MINISTÉRIO DA SAÚDE (BR). Secretaria de Gestão do Trabalho e da Educação em Saúde. Política de educação e desenvolvimento para o SUS: caminhos para a educação permanente em saúde - Polos de Educação Permanente em Saúde. Brasília: Ministério da Saúde, 2004.

OLIVEIRA, Fernanda Maria do Carmo Silveira Neves de. et al. Educação permanente e qualidade da assistência à saúde: aprendizagem significativa no trabalho da enfermagem. Aquichán, Bogotá, v.11, n.1, p. 48-65, 2011. Disponível em: http://www.scielo.org.co/scielo.php?script=sci= arttext\&pid=S165759972011000100005\&lng=en\&nrm=iso. Acesso em: 24 set. 2015.

PASCHOAL, Amarílis Schiavon. $\mathbf{O}$ discurso do enfermeiro sobre educação permanente no grupo focal. 2004. Dissertação (Mestrado em Enfermagem) - Setor de Ciências da Saúde, Universidade Federal do Paraná, Curitiba, 2004.

PEDUZZI, M. et. al. Atividades educativas de trabalhadores na atenção primária: concepções de educação permanente e de educação permanente em saúde em saúde presentes no cotidiano de Unidades Básicas de Saúde em São Paulo. Interface - Comunicação, Saúde e Educação, Botucatu, v.13, n.30, p.121-34, jul./set. 2009. Disponível em: $\leq$ http://www.scielo.br/scielo.php?pid=s1414-32832009000300011\&script=sci_arttext $>$. Acesso em: 15 jul. 2015.

PIO, C. Treinamento e desenvolvimento de pessoas: dois lados da mesma moeda. 2006. 1p.

RICHARDSON, R. J. Pesquisa social: métodos e técnicas. 3. ed. São Paulo: Atlas, 1999.

SCHONBERGER, R.J. World class manufacturing: the next decade. Nova York: Simon \& Schuster, 1996.

SILVA, Gizelda Monteiro da; SEIFFERT Otília Maria L. B. Educação continuada em enfermagem: uma proposta metodológica. Revista Brasileira de Enfermagem, Brasília, v.62, n.3 2009. Disponível em: http://www.scielo. br/scielo.php?pid=S0034-71672009000300005\&script=sci_arttext. Acesso em: 20 set. 2015. 
SILVA, Kênia Lara; SENA, Roseni Rosângela. A educação de enfermagem: buscando a formação crítico-reflexiva e as competências profissionais. Revista Latino-Americana de Enfermagem, Ribeirão Preto, v. 14, n. 5, set./out., 2006. Disponível em: <http://www.scielo.br/pdf/rlae/v14n5/pt_v14n5a18.pdf >. Acesso em: 20 set. 2015.

TACHIZAWA, Takeshy; FERREIRA, Victor Claudio Paradela; FORTUNA, Antônio Alfredo Mello. Gestão com pessoas: uma abordagem aplicada às estratégias de negócios. 2 ed. Rio de Janeiro: FGV, 2006. 